\title{
Attention risque d'explosion!?
}

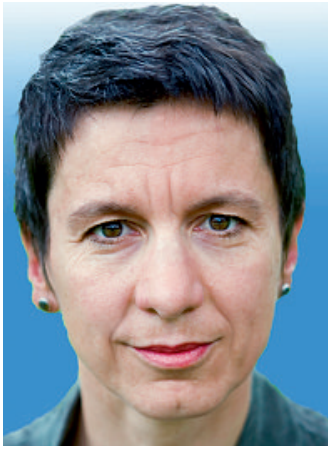

Anna Sax

L'article de Gerhard

Kocher à la page 504 traite également de la problématique des coûts de la santé.
* Anna Sax, lic. en économie, MHA, membre de la rédaction et copropriétaire et directrice de Tradig GmbH pour les analyses transdisciplinaires dans le domaine de la santé.
L'augmentation des dépenses de santé crée chaque année, et pendant plusieurs semaines, une certaine nervosité auprès des médias, du monde politique et des experts qui s'empressent de formuler des mesures d'économies: baisse des tarifs en vigueur, réduction des salaires, compression des coûts administratifs, amélioration de la prévention, jeu de la concurrence. Autrement dit, réformer de fond en comble le système de santé. Tous n'ont que le mot «économies» à la bouche pour autant bien sûr qu'elles concernent les autres. Qui ne sait plus que dire en appelle à la responsabilité individuelle des assurés. Tous s'affairent pour éviter le pire à savoir une explosion des coûts.

Mais si l'on y réfléchit un instant, on peut se demander ce qu'il y a de si grave à ce que les dépenses de santé augmentent de $3 \%$ en moyenne par année? Et pourquoi personne ne réagit vertement à l'augmentation des loyers ni ne se plaint que nous dépensions de plus en plus d'argent pour la mobilité et les loisirs? Les coûts des ménages en matière de téléphonie mobile, d'internet et de télévision augmentent de plus de $6 \%$ en moyenne depuis l'an 2000, soit plus du double des dépenses de santé. Pourquoi donc personne ne met en garde contre les risques d'explosion dans ce domaine? Et comment se fait-il que personne ne se réjouisse de la croissance d'un secteur économique qui génère à lui seul plus d'un demi-million de places de travail et contribue à l'amélioration de la qualité de vie de la population?

Tous les experts s'accordent à dire que la difficulté réside dans le fait que les dépenses de santé augmentent plus rapidement que celles des autres secteurs économiques privant ainsi p. ex. la formation ou la sécurité sociale de précieuses ressources. Or, cet argument n'est plus d'actualité aujourd'hui. En effet, depuis 2000 , les dépenses de santé évoluent au même rythme que l'économie et représentent une part constante du produit intérieur brut d'environ $10 \%$ à $11 \%$. Elles restent donc à un niveau élevé mais stable et c'est pourquoi on ne peut pas parler d'explosion des coûts.

En 2006, l'OCDE et l'OMS ont attesté de la haute qualité du système de santé suisse. Le statut sanitaire de la population suisse est l'un des meilleurs des pays de l'OCDE. Tout le monde a accès aux soins, l'éventail des prestations est large et la satisfaction des clients élevée. Mais les Suisses doivent payer le prix fort. Les experts de l'OCDE mentionnent en effet dans leur rapport que ces coûts élevés résultent certainement d'une décision de société. D'autres pays tels que la Finlande, l'Espagne ou la Grande-Bretagne atteignent avec moins d'argent des résultats similaires en matière de santé.
On dirait donc qu'il règne en Suisse un certain consensus sur le fait que nous voulons nous offrir ce luxe. Nous avons la plus grande liberté possible lors du choix du fournisseur de prestations, avons rapidement accès aux nouvelles technologies, n'avons pas de listes d'attentes digne de ce nom et sommes pris en charge par du personnel soignant hautement qualifié. Cela coûte certes très cher, mais voulons-nous véritablement renoncer à la qualité de ce système?

\section{Peut-on donc dire que tout va pour le mieux? La réponse n'est pas si simple}

Tout va pour le mieux alors? La réponse n'est pas aussi simple et il y a nécessité d'agir. Tous ceux qui œuvrent dans le domaine de la santé peuvent en dire long sur les structures inefficaces, les faux incitatifs, les doublons, le manque de coordination et l'argent gaspillé. Le potentiel d'optimisation est certes important, mais le principal problème ne concerne pas les coûts mais plutôt le financement. Car ce sont les primes d'assurance maladie qui augmentent plus que la moyenne et représentent une charge de plus en plus importante pour une grande partie de la population. Ce sont moins les dépenses de santé que la hausse des primes d'assurances qui touchent directement notre porte-monnaie. Si explosion il y a, alors c'est bien dans ce domaine-là.

Alors que les pouvoirs publics ont pu, au cours de ces dernières années réduire continuellement leur participation financière, la part incombant à l'assurance de base a en revanche augmenté. Cela est principalement dû au report des prestations hospitalières vers le secteur ambulatoire, un domaine où les assureurs répondent entièrement des frais. Le nouveau financement des hôpitaux va encore accentuer le phénomène de transfert du financement en préférant la formule d'une prime par tête à l'impôt progressif sur le revenu. La classe moyenne en fera les frais et ne pourra pas profiter des réductions de primes. Notre objectif principal ne devrait donc pas être d'économiser des coûts. Dans ce contexte, il paraît plus important de maintenir la qualité élevée des soins et de veiller à un financement durable et socialement tolérable du système de santé.

Anna Sax * 